\title{
Transient Management of Reconfigurable Digital Filters in Case of Sinusoidal Excitation
}

\author{
Tamás Kovácsházy, Gábor Péceli, László Sujbert \\ Department of Measurement and Information Systems \\ Budapest University of Technology and Economics, Hungary \\ Email: \{khazy,peceli,sujbert\}@mit.bme.hu
}

\begin{abstract}
Reconfigurable digital filters are promising solutions to filtering problems in hybrid systems, where digital filters with timeinvariant configuration are found to be inappropriate. However, reconfiguration of digital filters causes intermediate disturbances, reconfiguration transients that have to be minimized by proper transient management. In this paper the one-step reconfiguration method is analyzed for reconfigurable digital filters in case of sinusoidal excitation. First the energy function of the reconfiguration transient is formulated, and later analyzed. The implementation structure is identified as key factor influencing the energy of the reconfiguration transienst. Orthogonal implementation structures are proposed as the most suitable alternatives.
\end{abstract}

Keywords - Reconfigurable systems, hybrid systems, reconfiguration transients, transient reduction, transient management

\section{INTRODUCTION}

Reconfigurable digital filters play an important role in model-based, structurally adaptive systems $[1,2,3]$ such as complex measurement systems, industrial or vehicular control, sound and video processing [4], software radios, etc. In these systems, as the requirements and the environment change some control mechanisms modify the signal processing components run-time to fully meet the specified requirements. Unfortunately, however, due to reconfigurations transient phenomena might occur, which are to be minimized by proper design.

In [3] a new methodology was proposed to reduce the transient of reconfigurable systems for the case of whitenoise excitation, which utilizes the fact that, (1) the same transfer function can be realized by different processing structures, and (2) these alternative implementations show very different transient properties following reconfigurations.

In this paper the details of a similar study is presented for the case of sinusoidal excitation. A closed form expression of the reconfiguration transient energy function is elaborated and analyzed, possible transient reduction methods are derived, and the alternative implementations of transfer functions are also analyzed.

\section{A. Background}

The optimal transition after reconfiguration is highly dependent on the application; therefore, various transient definitions and transient measures exist. However, in most of the cases, the transient defined as

$$
f_{t r}(n)=f(n)-f_{i d}(n),
$$

where $f_{t r}(n)$ is the transient, $f(n)$ is the observed quantity in the investigated reconfigurable system, and $f_{i d}(n)$ is the same quantity observed in an ideal system. In addition, a transient measure is also required to compare the behavior of contending alternatives for transient reduction. The average energy of the transient, defined as

$$
\left\|f_{t r}\right\|_{2}^{2}=\sum_{n=-\infty}^{\infty}\left|f_{t r}(n)\right|^{2}
$$

can be used as a measure of selection in a large number of applications.

The input-output mappings of (linear) dynamic systems defined by their impulse response in the time-domain or by their transfer function in the frequency-domain do not specify how the internal processing is performed within the system. It is the state variable formulation

$$
\begin{gathered}
\mathbf{x}(n+1)=\mathbf{A x}(n)+\mathbf{B u}(n), \\
\mathbf{y}(n)=\mathbf{C x}(n)+\mathbf{D u}(n)
\end{gathered}
$$

which provides an appropriate framework to describe such systems, where the internal behavior considerably influences the overall performance. From now, we will consider only state-variable formulations that are minimal in the sense of state variables and have a nonsingular state transition matrix A with full eigenvector system. The transfer function of the represented system is invariant to the so-called similarity transformations

$$
(\mathbf{A}, \mathbf{B}, \mathbf{C}, \mathbf{D}) \rightarrow\left(\mathbf{T}^{-1} \mathbf{A T}, \mathbf{T}^{-1} \mathbf{B}, \mathbf{C T}, \mathbf{D}\right),
$$

where $\mathbf{T}$ is a nonsingular transformation matrix. Consequently there exist an infinite number of systems that realize the same transfer function. Various IIR filter structures $[5,6,7]$ have been developed, such as some of the Lattice structures and the orthogonal resonator-based structure, that utilize this invariance of the realized transfer function and to achieve certain advantages, e.g., better performance under finite word-length realizations. 


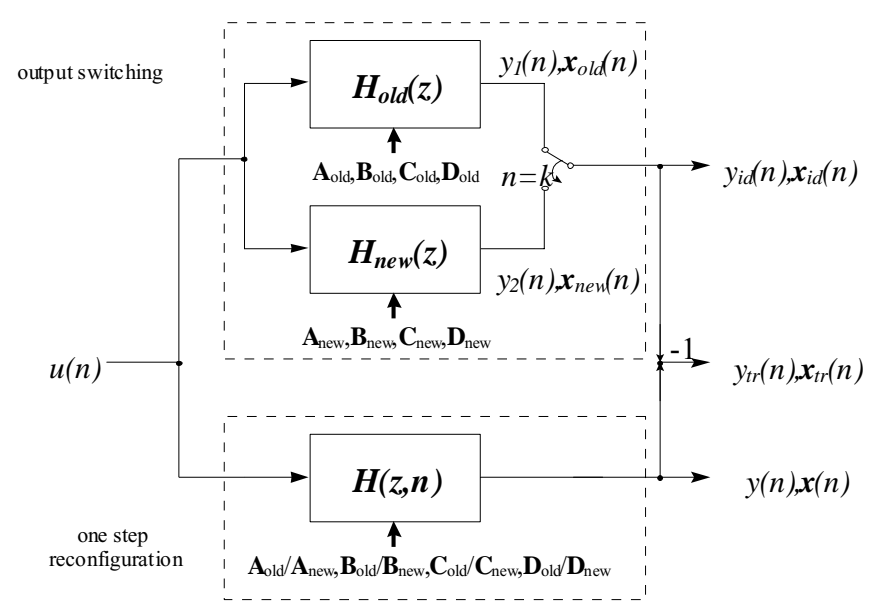

Figure 1. Experimental setup to investigate the reconfiguration transients.

\section{B. Experimental setup}

In our investigations we consider the transients of a onestep reconfiguration at $n=k$. The one-step reconfiguration is in the center of our interest, because it is used in various application, it is easy to implement, and requires minimal resources. We compare the transients produced by the tested systems to the output of the output switching method [2]. The experimental setup is shown in Figure 1.

We assume that before the reconfiguration all the examined components, i.e., the system to be reconfigured, represented by a time-varying transfer function $H(n, z)$, the "old" system, represented by $H_{\text {old }}(z)$, and the "new" system, represented by $H_{\text {new }}(z)$, are in steady-state, or at least very close to that. After reconfiguration, however, the state variables inherited from the old configuration $[H(n, z)$ with $\boldsymbol{A}_{\text {old }}, \boldsymbol{B}_{\text {old }}, \boldsymbol{C}_{\text {old }}$, and $\left.\boldsymbol{D}_{\text {old }}\right]$ are not necessarily steady state values for the new configuration $\left[H(n, z)\right.$ with $\boldsymbol{A}_{\text {new }}, \boldsymbol{B}_{\text {new }}, \boldsymbol{C}_{\text {new }}$, and $\left.\boldsymbol{D}_{n e w}\right]$. The transient of the state variable vector $\mathbf{x}_{t r}(k)$ is the difference of the state variable vector of $H_{\text {new }}(z)$ and the state variable vector of $H(n, z)$, which is identical to the state variable vector of $H_{\text {old }}(z)$. Therefore, the transient of the state variable vector in the system reconfigured by the one-step reconfiguration can be expressed as

$$
\mathbf{x}_{\text {tr }}(k)=\mathbf{x}_{\text {old }}(k)-\mathbf{x}_{\text {new }}(k),
$$

and the output transient can be computed as

$$
\mathbf{y}_{t r}(n)= \begin{cases}0, & \text { for } 0 \leq n<k \\ \mathbf{C}_{n e w} \mathbf{A}_{n e w}^{n-k} \mathbf{x}_{t r}(k), & \text { for } n \geq k\end{cases}
$$

\section{ANALYTIC APPROACH}

We consider a single sinusoid as input in the form of

$$
u(n)=U \cos (\Theta n+\varphi),
$$

where $U, \Theta$ and $\varphi$ stand for the amplitude, frequency and phase, respectively. Using the complex notation $u(\mathrm{n})=$ $\operatorname{Re}\left(\bar{U} e^{j \Theta n}\right)$, where $\bar{U}=U e^{j \varphi}$ is the complex amplitude.

The steady-state behavior of linear, time-invariant and discrete-time systems can be examined using its transfer characteristics $\bar{H}=H e^{j \rho}$ and the complex amplitude $\bar{U}=U e^{j \varphi}$ of the input. The complex amplitude of the output can be expressed as

$$
\bar{Y}=\bar{H} \bar{U}=H e^{j \rho} U e^{j \varphi},
$$

$H$ and $\rho$ are functions of $\Theta$, however, this is indicated only in case of possible misunderstanding. The output samples can be given as

$$
u(n)=\operatorname{Re}\left(\bar{Y} e^{j \Theta n}\right)=H U \cos (\rho+\varphi+\Theta n) .
$$

For the approach presented in Figure 1 we can derive the transfer values from the input to the state variables $x_{i}$ at the discrete frequency $\Theta: \bar{H}_{o, x_{i}}$ for the old and $\bar{H}_{n, x_{i}}$ for the new configuration. The transfer value responsible for the transient from the input to the state variables $x_{i}$ at the discrete frequency $\Theta: \bar{H}_{t r, x_{i}}$ is exactly the difference of the difference of the two previous transfer values:

$$
\bar{H}_{t r, x_{i}}=\bar{H}_{\text {old }, x_{i}}-\bar{H}_{\text {new }, x_{i}} .
$$

Using (10) and (9) the transient state $x_{t r, i}(k)$ can be expressed as:

$$
x_{t r, i}(k)=H_{t r, i} U \cos \left(\rho_{t r, i}+\varphi+\Theta k\right) .
$$

The energy of the output transient can be computed after some simplifications as show in (12), where $w_{i j}$ the element of the observability Grammian computed in the form of

$$
\mathbf{W}=\sum_{l=0}^{\infty}\left(\mathbf{C A}^{l}\right)^{\mathrm{T}}\left(\mathbf{C} \mathbf{A}^{l}\right)=\mathbf{A}^{T} \mathbf{W A}+\mathbf{C} \mathbf{C}^{T},
$$

and $N$ is the order of the filter. 
Utilizing equality $2 \cos A \cos B=\cos (A-B)+\cos (A+B)$, where $A=\rho_{t r, i}+\varphi+\Theta k$ and $B=\rho_{t r, j}+\varphi+\Theta k$ we get the (14). Equation (14) can be decomposed into two parts, resulting (15). The first part of (15) is independent of $\varphi$, and $k$, while second is not. In other words, the transient energy function (16) for a given reconfigurable system on a given frequency has a phase independent constant component $\left(\mathrm{C}_{0}\right)$, and a phase dependent sinusoidal component $\left(\mathrm{C}_{1}\right.$ amplitude, $\delta$ relative phase to the input phase). The frequency of this sinusoidal component is doubled, and $\mathrm{C}_{1} \leq \mathrm{C}_{0}$, because energy functions are strictly non-negative.

\section{TRANSIENT REDUCTION}

The analyses of (15) and (16) shows that there are two possible approaches to reduce reconfiguration transients in case of the applied one-step reconfiguration.

As the first approach, assuming sinusoidal input with given frequency and phase, we might reduce transient energy by properly setting, i.e., delaying, the reconfiguration to a more appropriate later time. Unfortunately, the determination of this time instant in practical applications requires intensive run-time calculations and its effectiveness depends on the relative size of the constant and phase dependent components.

Experiments show that for orthogonal filter structures $[5,6,7] \mathrm{C}_{1}<<\mathrm{C}_{0}$ by orders of magnitudes most cases for the investigated transfer functions. There is no theoretical background elaborated to explain this behavior; however, for the all-pass output of these structures, which all orthogonal structures have as a common feature, $\mathrm{C}_{1}=0$. This special property is explained by the fact that the observability Grammian is unity matrix for the all-pass output, and the internal state variables, and therefore the $\bar{H}_{t r, i}$ terms also, are orthogonal canceling out the sinusoidal components in the expression of the transient energy function. This preferable cancellation property is only partial, resulting $\mathrm{C}_{1}<<\mathrm{C}_{0}$, for the arbitrary transfer function output. However, $\mathrm{C}_{1}$ is comparable to $\mathrm{C}_{0}$ for the widely used direct structures and the state-space implementation provided by Matlab for the tested transfer functions.

As a second approach, worst-case transients can be reduced overall by using orthogonal structures. Experiments with the investigated transfer functions show that reconfigurable filters implemented using orthogonal structures generate orders of magnitudes smaller reconfiguration transients than the classic direct structures, or other non-orthogonal implementations for sinusoidal excitation. The orthogonal structures generate smaller reconfiguration transient than the non-orthogonal ones because their internal state variables, as it has been already noted previously, orthogonal and inherently scaled. For sinusoidal excitation the state variable moves on a circle, and the radius of this circle depends on the amplitude of the excitation only, and does not depend on the implemented transfer function nor the frequency or the phase of the excitation, which limits the maximum $\boldsymbol{x}_{\mathrm{tr}}(\mathrm{k})$; and based on (6) the output transient also.

Real signals are never pure sinusoidal; however, most cases a sinusoidal and additive white noise can model real signals appropriately for theoretical analysis. In [3] a similar discussion of reconfiguration transients for pure white-noise excitation is provided, which also proposes orthogonal structures to reduce reconfiguration transients. Initial simulation data shows that orthogonal structures are better even for such complex signals; however, there has been no theoretical background developed yet to explain the observed behavior.

\section{ILLUSTRATIVE EXAMPLE}

During the experiments the expression of the output transient (15) has been evaluated and compared to simulations for a relatively large number of IIR filter specification sets and various filter structures such as the normalized lattice [7], orthogonal resonator-based [6], statespace implementation (as the matrixes are provided by the Signal Processing Toolbox of Matlab), and direct form transposed II (direct implementation of the transfer function). The simulations require substantially more time, typically 10 times or more, to execute than the expression of (15).

As an example, a $6^{\text {th }}$ order pass-band Butterworth IIR filter

$$
\begin{gathered}
\sum_{l=0}^{\infty} y_{t r}^{2}(l)=\sum_{j=0}^{N} \sum_{i=0}^{N} w_{n, i j} H_{t r, i} H_{t r, j} U^{2} \cos \left(\rho_{t r, i}+\varphi+\Theta k\right) \cos \left(\rho_{t r, j}+\varphi+\Theta k\right) \\
\sum_{l=0}^{\infty} y_{t r}^{2}(l)=\sum_{j=0}^{N} \sum_{i=0}^{N} w_{n, i j} H_{t r, i} H_{t r, j} U^{2} \frac{1}{2}\left(\cos \left(\rho_{t r, i}-\rho_{t r, j}\right)+\cos \left(\rho_{t r, i}+\rho_{t r, j}+2 \varphi+2 \Theta k\right)\right) \\
\sum_{l=0}^{\infty} y_{t r}^{2}(l)=\sum_{j=0}^{N} \sum_{i=0}^{N} w_{n, i j} H_{t r, i} H_{t r, j} U^{2} \frac{1}{2} \cos \left(\rho_{t r, i}-\rho_{t r, j}\right)+ \\
+\sum_{j=0}^{N} \sum_{i=0}^{N} w_{n, i j} H_{t r, i} H_{t r, j} U^{2} \frac{1}{2} \cos \left(\rho_{t r, i}+\rho_{t r, j}+2 \varphi+2 \Theta k\right) \\
\sum_{l=0}^{\infty} y_{t r}^{2}(l)=C_{0}+C_{1} \cos (\delta+2 \Theta k)
\end{gathered}
$$



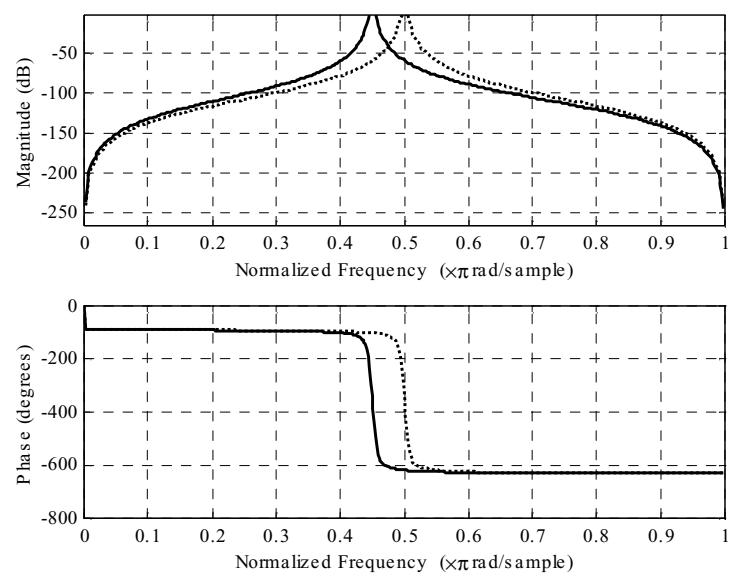

Figure 2. The frequency response of the filters used during the experiments

is used for demonstration, during the reconfigurations the center frequency of the filter is changed between $0.5 f_{s} / 2$ and $0.45 f_{s}, / 2$, see Figure 2. for frequency response of the filters.

The transients are investigated as the function of frequency and phase of the input sinusoid. The maximum transient energies are plotted on Figure 3. as the function of the input sinusoid frequency. The normalized lattice and resonator-based structures, which are both orthogonal [5], produce the smallest worst-case transients (the smallest transients in the whole frequency range) and in all other experiments very similar results are observed.

\section{V.CONCLUSIONS}

The reconfiguration transients of reconfigurable digital filers using the one-step reconfiguration analyzed in case of sinusoidal excitation. The analyzes of the elaborated reconfiguration transient energy function shows that, (1) orthogonal structure based implementations allow reduction of the reconfiguration transients dramatically compared to classic implementation structures such as the direct structure, (2) the amplitude of the sinusoidal component of the reconfiguration transient energy function is small compared to the constant component for the orthogonal structure based implementation, which makes the proposed delayed reconfiguration based transient management method unfeasible for these structures. However, for other implementation structures this transient reduction scheme may be useful.

\section{ACKNOWLEDGEMENTS}

This research was sponsored by DARPA (US) (F3361599-C-3611) and by the Hungarian Ministry of Education (OM-FKFP 0654/2000).

\section{REFERENCES}

[1] Grossman, R.L., A. Nerode, A.P. Ravn, and H. Rischel, Eds., Hybrid Systems, Lecture Notes in Computer Science, no. 736, New York: Springer Verlag, 1993.

[2] Sztipanovits, J., D.M. Wilkes, G. Karsai, Cs. Biegl, L.E. Lynd, "The Multigraph and structural adaptivity," IEEE Transactions on Signal Processing, Vol.41, No.8, pp. 2695-2716, Aug. 1993.

[3] Tamás Kovácsházy, Gábor Péceli, Gyula Simon, "Transients in Reconfigurable Signal Processing Channels," IEEE Trans. on Instrumentation and Measurement, Vol. 50., pp. 936-940, August, 2001.

[4] Valimaki, V., T.I. Laakso, "Suppression of transients in variable recursive filters with a novel and efficient cancellation method," IEEE Trans. on Signal Processing, Vol. 46, (1998), pp. 3408-3414.

[5] Roberts, R.A., C.T. Mullis, Digital Signal Processing. New York: Addison-Wesley, 1987.

[6] Padmanabhan, M., K. Martin, and G. Péceli, Feedback-based Orthogonal Filters: Theory, Applications, and Implementation. Kluwer Academic Publishers, Boston-Dordrecht-London, 1996. 265 p.

[7] J. Chung, and Keshab K. Parhi, Pipelined Lattice and Wave Digital Filters. Kluwer Academic Publishers, 1996

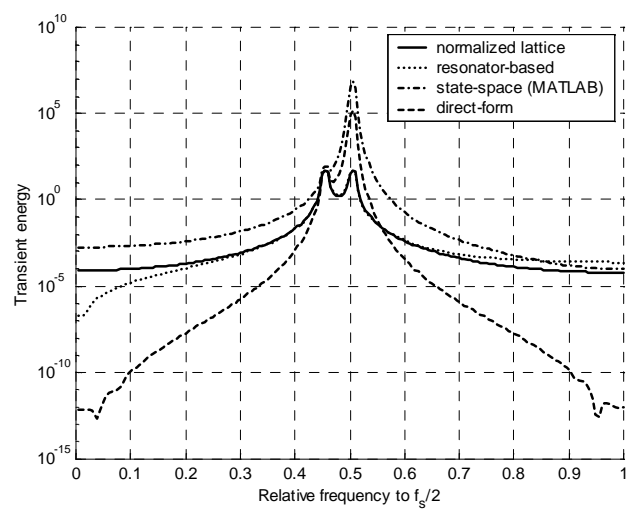

(b)

(a)

Figure 3. Transient energies as the function of input frequency for various filter structures (a) decreasing the center frequency (b) increasing the center frequency 